\title{
Zar Theorie der Gamma-Functionen.
}

Von

\author{
Alfred Pringsheim in München.
}

Die Theorie derjenigen Transcendenten, welche man heute zumeist als Gamma-Functionen (sonst auch als Analytische Facultäten oder $\Pi$-Functionen) bezeichnet, lässt sich entweder nach den Methoden der älteren Analysis auf der Grundlage eines definirenden analytischen Ausdruckes oder - mehr im Sinne der neueren Functionentheorie mit Benutzung von definirenden Functionalgleichungen und allgemeinen Functionaleigenschaften aufbauen. Sieht man von dieser letzteren Methode hier $a b^{*}$ ), so bieten sich als Ausgangspunkt noch zwei

*) Zu den verhältnissmässig wenig zahlreichen Arbeiten, welche diese functionentheoretische Methode befolgen, gehört ausser der berühmten Abhandlung des Herrn Weierstrass über die „Analytischen Facultäten" (Crelle's Journal, Bd. 51) ein sehr beachtenswerther Aufsatz von Herrn Prym: "Zur Theorie der Gamma-Funetionen" (Crelle's Journal, Bd. 82, S. 165 ff.); ferner die Hankel'sche Habilitations-Schrift: „Die Euler'schen Integrale bei unbeschränkter Variabilität des Arguments" (Leipzig 1863), welche jedoch zum Theil auf falschen Praemissen beruht and in Folge dessen in ihren Hauptresultaten hinfällig ist. Der Grund. fehler des Ganzen liegt in der Benutzung des folgenden Satzes zum Beweise der eindeutigen Bestrmmtheit von $\Gamma(x)$ durch gewisse Grundeigenschaften:

"Eine monodrome, stetige, für alle endlichen Werthe des Argumentes von Null und Unendlich verschiedene Function von $x$, die den Gleichungen $\varphi(x+1)=\varphi(x)$ $\varphi(0)=1$ genügt und auf einer endlichen Strecke der reellen Axe reell bleibt, ist eine Constante $\varphi(x)=1 . "$ (a.a. O. S.15.16). Dieser Satz ist falsch: den Bedingungen desselben genügt nämlich offenbar jede Exponentialgrösse der Form: $\varphi(x)=e^{\psi(x)}$, wo $\psi(0)=0$ und $\psi(x)$ in übrigen eine monodrome, im Endlichen stets endliche und stetige Function bedeutet, die für reelle $x$ selbst reell ist und die Periode 1 besitzt - wie z. B. $\psi(x)=G(\sin 2 \pi x)$, wo $G(x)$ eine algebraische oder transcendente ganze Function ohne constantes Glied bezeichnet. - Soll der obige Satz richtig sein, so muss zu den angegebenen Voraussetzungen noch die hinzukommen, dass auch $\varphi(\infty)$ endlich und bestimmt sein soll: hierdurch verliert aber dann der Satz vollständig seine Brauchbarkeit für den vorliegenden Zweck. Hankel, der seinen Irrthum später selbst erkannt hat, gesteht dies auch ausdrücklich zu. (Schlömilch, Zeitschr. für Math. Jahrg. IX, S. 5). Dagegen hat es keinerlei Schwierigkeit, das Hankel'sche Verfahren mit Benützung derjenigen Principien zu rectificiren, welche Herr Weierstrass in seiner Abhandlung: "Zur Theorie der eindeutigen analytischen Functionen" (Abh. der Berl. Academie 1876) gegeben hat. 
fundamental verschiedene Definitionen dar: die (beiläufig bemerkt, um ein Jahr ältere) Gauss'sche durch ein schon bei Euler vorkommendes unendliches Product*) und die Legendre'sche durch ein ebenfalls von Euler herrührendes bestimmtes Integral **). Hat nun auch die Gauss'sche Definition auf die oben erwähnte functionentheoretische Behandlung unserer Transcendenten zweifellos einen sehr wesentlichen Einfluss ausgeäbt, so ist in der gesammten übrigen, äusserst umfangreichen Literatur über diesen Gegenstand die Legendre'sche Definition nicht nur bevorzugt, sondern - mit verschwindenden Ausnahmen $* * * *$ ) - geradezu ausschliesslich acceptirt worden; ja es scheint sogar, dass Gauss selbst späterhin die Integraldefinition als zweckmässiger erachtet hat: wenigstens enthält das in seinem Nachlasse vorgefundene gedruckte Handexemplar der Abhandlung Disquisitiones generales circa seriem etc., wie Herr Schering als Herausgeber des bezüglichen Nachlasses mittheilt $\dagger$ ), die ausdrückliche Aufzeichnung: „Die beste Definition von $\Pi m$ ist, dass

$$
\Pi m=\int_{-\infty}^{+\infty} e^{(n+1) x} e^{-e^{x}} d x .
$$

(NB. Dieselbe geht aus der entsprechenden Legendre'schen

$$
\int_{0}^{1}\left(\lg \frac{1}{x}\right)^{m} d x
$$

hervor, wenn man $e^{-e^{x}}$ an Stelle der Integr.-Variablen $x$ einführt.)

Der Grund dieser Erscheinung dürfte darin zu suchen sein, dass die wesentlichste Bedeutung der $\Gamma$-Functionen in ihren mannigfaltigen

*) Euler giebt das betreffende Product als Lösung der Aufgabe, die Reihe 1, 1.2, 1.2.3,... zu interpoliren, d. h. also als Verallgemeinerung von $z$ ! für andere als ganzzahlige positive Werthe von $n-$ und zwar im Cap. XVII seiner Instit. Calc. diff. (S. 834); bei Gauss erscheint es dann als Definition einer neuen Transcendenten $\Pi(z)$ (welche mit dem Legendre'schen $\Gamma(z+1)$ identisch ist) in der 1813 publicirten Abhandlung: Disquisitiones generales circa seriem etc. (Ges. W. Bd. III, S. 145, Art. 20).

**) Legendre definirt das von Euler lediglich für rationale Werthe von $a$ behandelte Integral $\int_{0}^{1}\left(\lg \frac{1}{x}\right)^{a-1} d x$ für beliebige positive a als eine Function der reellen Variablen $a$, welche er mit $\Gamma(a)$ bezeichnet: zum ersten Male in dem 1814 veröffentlichten $4^{\text {ten }}$ Theile seiner Exercices de Calcul intégral (T. II, p. 4)

***) Trotz der grossen Zahl von Abhandlungen und Compendien, welche ich über diesen Gegenstand consultirt habe, ist mir nur ein kleiner Aufsatz des Herrn Liouville (Journal de Mathématiques, T. XVII p. 448) begegnet, welcher von der Gauss'schen Definition ausgeht (a. a. 0. p. 451).

t) Ges. W. Bd. III, S. 230. 
Beziehungen zur Integralrechnung und deren unmittelbaren Anwendungen liegt. Auf der anderen Seite ist aber nicht zu verkennen, dass die Gauss'sche Definition nicht pur den Vorzug grösserer Allgemeinheit besitzt (wegen der Möglichkeit, der Variablen alle möglichen reellen und complexen Werthe beizulegen), sondern - was noch stärker in's Gewicht fällt - dass sie auch gestattet, die gesammte Theorie weit kürzer und einheitlicher darzustellen, als dies mit den Mitteln der Integralrechnung allein möglich ist: an die Stelle einer Reihe von Kunstgriffen, durch welche die einzelnen Sätze der Theorie völlig unvermittelt neben einander gestellt werden, tritt bei consequenter Anwendung der Productdefinition eine allgemeine und durchsichtige Methode*).

Wägt man auf diese Weise die Vorzüge jener beiden Definitionen gegen einander $a b$, so dürfte vom didaktischen Standpunkt es sich am meisten empfehlen, zwar von der Integraldefinition auszugehen, sodann aber vor allem die Productentwicklung daraus abzuleiten und nunmehr die übrige Theorie auf den gewonnenen Productausdruck zu gründen. Obschon dieser Gedanke keinerlei Anspruch auf Neuheit erhebt, so ist derselbe, glaube ich, bisher nirgends mit hinreichender Consequenz durchgeführt worden; namentlich aber scheint mir von den bisher üblichen Methoden, die unendliche Productentwicklung für $\Gamma(x)$ aus der Integraldefinition herzuleiten, keine einzige mit vollkommener Natürlichkeit aus der Integraldefinition herauszuwachsen und jenes Ziel ohne Umwege zu erreichen. Ich hoffe nun, dass von den beiden bezüglichen Methoden, welche ich im Folgenden mittheile ( 11 und $\S 3$ ), besonders die erste den obigen Anforderungen möglichst entspricht, während die zweite als erhebliche Abkürzung einer bisher vielfach angewendeten, aber etwas umständlichen Herleitungsweise vielleicht ebenfalls nicht ganz ohne Interesse erscheinen mag. An die erste Methode knüpfe ich zugleich die Ableitung der wichtigeren Sätze und Formeln in übersichtlicher und den neueren analytischen Anschauungen möglichst Rechnung tragender Darstellung (\$§ 1,2). Schliesslich wird die im $\S 1$ zur Entwicklung von $\Gamma(x)$ benutzte Methode auch auf das Euler'sche Integral erster Gattung angewendet und dessen Zusammenhang mit der Gamma-Function daraus hergeleitet (\$ 4). -

*) Damit soll keineswegs gesagt sein, dass die betreffenden, lediglich auf Integraltransformationen etc. gegründeten Beweise der theoretischen Berechtigung und des Interesses entbehren: nur erscheinen dieselben bei weitem künstlicher und besitzen in Folge dessen auch geringere Ueberzeugungskraft. 
$\S 1$.

Erste Methode zur Entwicklung der Г-Function in ein unendliches

Product und Ableitung der daraus resultirenden Hauptformeln.

Die $\Gamma$-Function (das Euler'sche Integral zweiter Gattung) wird definirt durch die Gleichung:

$$
\left.\Gamma(x)=\int_{0}^{\infty} e^{-\alpha} \alpha^{x-1} d \alpha^{*}\right)
$$

und ist hiernach für jedes endliche $x>0$ eine eindeutige, endliche und stetige Function ihres Arguments. Durch partielle Integration ergiebt sich:

$$
\Gamma(x)=\left[\frac{e^{-\alpha} \cdot \alpha^{x}}{x}\right]_{0}^{\infty}+\frac{1}{x} \int_{0}^{\infty} e^{-\alpha} \alpha^{x} d \alpha=\frac{1}{x} \Gamma(x+1)
$$

oder:

$$
\Gamma(x+1)=x \Gamma(x)
$$

und durch $n$ malige Anwendung dieser Recursionsformel:

(3) $\Gamma(x+n)=x(x+1) \cdots(x+n-1) \Gamma(x)=\prod_{1}^{n} \Gamma(x+\nu-1) \cdot \Gamma(x)$, woraus speciell für $x=1$ folgt:

$$
\Gamma(n+1)=1 \cdot 2 \cdots n-\mathrm{da}: \Gamma(1)=\int_{0}^{\infty} e^{-\alpha} d \alpha=1 .
$$

Setzt man die Gleichung (3) in die Form

$$
\Gamma(x)=\frac{1}{x(x+1) \cdots(x+n-1)} \Gamma(x+n),
$$

(wo also $n$ jede beliebig grosse ganze positive Zahl bedeutet), so wird dieselbe offenbar dazu benützt werden können, $\Gamma(x)$ durch ein unendliches Product darzustellen, sobald es gelingt $\Gamma(x+n)$ für $n=\infty$ durch bekannte Functionen von $n$ darzustellen und sodann nachzuweisen, dass die rechte Seite der Gl. (5) für $n=\infty$ convergirt. Dabei genügt es, die positive Grösse $x$ zunächst $<1$ anzunehmen, da $\Gamma(1)$

*) Diese Form des Integrals, welche aus der bei Legendre ausschliesslich vorkommenden:

$$
\Gamma(x)=\int_{0}^{1}\left(\lg \frac{1}{\beta}\right)^{x-1} d \beta
$$

durch die Substitution $\beta=e^{-\alpha}$ hervorgeht, findet sich, soviel ich feststellen konnte, zum ersten Mal bei Poisson: Mémoire sur les Intégrales définies etc. (Journal de l'Ecole polyt. Cah. 19, p. 477). 
bereits bestimmt ist, und die Formel (3) lehrt, wie man jedes Argament, das $>1$ ist, auf ein solches zwischen 0 und 1 reduciren kann. Zerlegt man $\Gamma(x+n)$ folgendermassen:

$$
\Gamma(x+n)=\int_{0}^{n} e^{-\alpha} \alpha^{n-1} \alpha^{x} d \alpha+\int_{n}^{\infty} e^{-\alpha} \alpha^{n-1} \alpha^{x} d \alpha,
$$

so hat man in ersten Theilintegrale $\alpha^{x}<n^{x}$, dagegen $\alpha^{x}>\alpha \cdot n^{-1+x}$ (letr.teres, weil $x$ ein pos. ächter Bruch und daher für $\left.\alpha<n:\left(\frac{\alpha}{n}\right)^{x}>\frac{\alpha}{n}\right)$; dagegen im zweiten Theilintegrale $\alpha^{x}<\alpha \cdot n^{-1+x}$ und $\alpha^{x}>n^{x}$. In Folge dessen erhält man an Stelle der obigen Gleichung die beiden Ungleichungen

$$
\Gamma(x+n)\left\{\begin{array}{l}
<n^{x} \int_{0}^{n} e^{-\alpha} \alpha^{n-1} d \alpha+n^{-1+x} \int_{n}^{\infty} e^{-\alpha} \alpha^{n} d \alpha \\
>n^{-1+x} \int_{0}^{\infty} e^{-\alpha} \alpha^{n} d \alpha+n^{x} \int_{n}^{\infty} e^{-\alpha} \alpha^{n-1} d \alpha,
\end{array}\right.
$$

oder:

$$
\frac{\Gamma(x+n)}{n^{x}}\left\{\begin{array}{l}
<\frac{1}{n} \int_{0}^{\infty} e^{-\alpha} \alpha^{n} d \alpha+\int_{0}^{n} e^{-\alpha} \alpha^{n-1} d \alpha-\frac{1}{n} \int_{0}^{n} e^{-\alpha} \alpha^{n} d \alpha, \\
>\int_{0}^{\infty} e^{-\alpha} \alpha^{n-1} d \alpha-\int_{0}^{n} e^{-\alpha} \alpha^{n-1} d \alpha+\frac{1}{n} \int_{0}^{n} e^{-\alpha} \alpha^{n} d \alpha .
\end{array}\right.
$$

Nun ist

$$
\begin{aligned}
& \int_{0}^{\infty} e^{-\alpha} \alpha^{n-1} d \alpha=\frac{1}{n} \int_{0}^{\infty} e^{-\alpha} \alpha^{n} d \alpha=\Gamma(n)=(n-1) ! \\
& \int_{0}^{n} e^{-\alpha} \alpha^{n-1} d \alpha=\left[\frac{e^{-\alpha} \alpha^{n}}{n}\right]_{0}^{n}+\frac{1}{n} \int_{0}^{n} e^{-\alpha} \alpha^{n} d \alpha,
\end{aligned}
$$

also:

$$
\int_{0}^{n} e^{-\alpha} a^{n-1} d \alpha-\frac{1}{n} \int_{0}^{n} e^{-\alpha} \alpha^{n} d \alpha=\frac{n^{n-1}}{e^{n}}
$$

und daher:

$$
\frac{\Gamma(x+n)}{n^{x}}\left\{\begin{array}{l}
<(n-1) !+\frac{n^{n-1}}{e^{n}} \\
>(n-1) !-\frac{n^{n-1}}{e^{n}}
\end{array}\right.
$$

sodass man setzen kann 


$$
\Gamma(x+n)=(n-1) ! n^{x}\left\{1+\vartheta \varepsilon_{n}\right\}
$$

wo $\vartheta$ einen positiven oder negativen ächten Bruch bedeutet und die Grösse

$$
\varepsilon_{n}=\frac{n^{n-1}}{(n-1) ! e^{n}}=\frac{n^{n}}{n ! e^{n}},
$$

wie sich leicht zeigen lässt, für $n=\infty$ verschwindet*). Man hat nämlich

$$
e^{n}=\sum_{0}^{\infty} \frac{n^{v}}{\nu !}>\frac{n^{n}}{n !}+\frac{n^{n+1}}{(n+1) !}+\cdots+\frac{n^{n+k}}{(n+k) !}+\cdots,
$$

also

wo

$$
(n-1) ! e^{n}>n^{n-1} \sigma_{n},
$$

$$
\sigma_{n}=1+\frac{n}{n+1}+\cdots+\frac{n^{k}}{(n+1) \cdots(n+k)}+\cdots
$$

d. h.:

$$
\frac{n^{n-1}}{(n-1) ! e^{n}}=\varepsilon_{n}<\frac{1}{\sigma_{n}} \text {. }
$$

Andererseits ist aber für jedes noch so grosse $k$

$$
\sigma_{n}>k \frac{n^{k}}{(n+1) \cdots(n+k)}>k \cdot \frac{1}{\left(1+\frac{k}{n}\right)^{k}}
$$

und somit, wenn man zunächst $k$ beliebig gross und sodann $n \geqq k^{2}$, also $\frac{1}{k} \geqq \frac{k}{n}$, annimmt:

$$
\sigma_{n}>k \cdot \frac{1}{\left(1+\frac{1}{k}\right)^{k}}>\frac{k}{e},
$$

*) Ich bemerke beiläufig, dass der ganz ähnlich gebildete Ausdruck

$$
\xi_{n}=\frac{n^{n}}{n ! 2^{n}}
$$

bei dem also lediglich an Stelle von $e=2,718 \ldots$ die Zahl 2 tritt, mit $n=\infty$ nicht verschwindet, sondern unendlich gross wird. Man hat nämlich

also

$$
k(n+1-k)<\left(\frac{n+1}{2}\right)^{2} \quad\left(1 \leqq k \leqq \frac{n}{2}\right)
$$

d. h.

$$
\begin{aligned}
(n-1) ! & =(2 \cdot \overline{n-1})(3 \cdot \overline{n-2}) \cdots \\
& <\left(\frac{n+1}{2}\right)^{n-2} \\
n ! 2^{n} & <4(n+1)^{n-1} \\
\xi_{n} & >\frac{1}{4}\left(\frac{n}{n+1}\right)^{n}(n+1)>\frac{n+1}{4 e}
\end{aligned}
$$

$$
\zeta_{\infty}=\infty \text {. }
$$


wird also mit $k \leqq \sqrt{n}$ beliebig gross, sodass in der That $\lim \sigma_{n}=\infty$ und somit

$$
\lim \varepsilon_{n}=0 \text { (für } n=\infty \text { ). }
$$

Mit Berücksichtigung von Gl. $(6)^{*}$ ) geht nun die Formel (5) in die folgende über:

$$
\Gamma(x)=\frac{1 \cdot 2 \cdots n-1}{x \cdot(x+1) \cdots(x+n-1)} n^{x}\left(1+\vartheta \varepsilon_{n}\right) \quad(-1<\vartheta<+1),
$$

in welcher $n$ beliebig gross genommen werden darf, während $\varepsilon_{\eta}$ durch Wahl hinlänglich grosser $n$ beliebig klein gemacht werden kann. Lässt man jetzt $n$ in's Unendliche wachsen, so wird

$$
\Gamma(x)=\frac{1}{x} \lim \frac{1 \cdot 2 \cdots n-1}{(x+1)(x+2) \cdots(x+n-1)} n^{x} \quad(n=\infty),
$$

oder indem man bemerkt, dass

$$
n^{x}=\left(\frac{2}{1} \cdot \frac{3}{2} \cdots \frac{n}{n-1}\right)^{x}=\left(1+\frac{1}{1}\right)^{x}\left(1+\frac{1}{2}\right)^{x} \cdots\left(1+\frac{1}{n-1}\right)^{x}
$$

gesetzt werden kann:

(9) $\Gamma(x)=\frac{1}{x} \lim _{n=\infty} \frac{\left(1+\frac{1}{1}\right)^{x}\left(1+\frac{1}{2}\right)^{x} \cdots\left(1+\frac{A}{n-1}\right)^{x}}{\left(1+\frac{x}{1}\right)\left(1+\frac{x}{2}\right) \cdots\left(1+\frac{x}{n-1}\right)}=\frac{1}{x} \prod_{1}^{\infty} \Gamma \frac{\left(1+\frac{1}{\nu}\right)^{x}}{1+\frac{x}{\nu}}$,

sofern dieses unendliche Product convergirt. Dass dies in der That der Fall ist - und zwar für jedes reelle oder complexe $x$ mit Ausschluss der Null und der ganzen negativen Zahlen**) - ergiebt sich am leichtesten, wenn man bildet:

(10) $\lg \Gamma(x)=-\lg x+\sum_{1}^{\infty}\left\{x \lg \left(1+\frac{1}{\nu}\right)-\lg \left(1+\frac{x}{\nu}\right)\right\}$,
(10a) $=-\lg x-x \sum_{1}^{\infty}\left\{\frac{1}{\nu}-\lg \left(1+\frac{1}{\nu}\right)\right\}+\sum_{1}^{\infty}\left\{\frac{x}{\nu}-\lg \left(1+\frac{x}{\nu}\right)\right\}$.

*) Schreibt man GL. (6) folgendermassen:

$$
\lim \frac{\Gamma(x+n)}{(n-1) ! n^{x}}=1
$$

so bestimmt dieselbe mit der Gleichung

$$
\Gamma(x+1)=x \cdot \Gamma(x)
$$

zusammen offenbar die $\Gamma$-Function vollständig, denn man erhalt aus diesen beiden Gleichungen ohne Weiteres die Product-Entwickelnng (8). In dem oben citirten Aufsatze des Herrn Prym wird dieser Satz in etwas verallgemeinerter Form auf functionentheoretischem Wege bewiesen.

*) Für verschwindende oder ganzzahlige negative Werthe von $x$ wird ein Factor des Productes unendlich, während das Product im übrigen convergent bleibt. 
Da nuu für $|x|<v$

$$
\frac{x}{v}-\lg \left(1+\frac{x}{\nu}\right)=\frac{1}{2} \cdot \frac{x^{2}}{\nu^{2}}-\frac{1}{3} \frac{x^{3}}{\nu^{3}}+\frac{1}{4} \frac{x^{4}}{v^{4}}-\cdots,
$$

also

$$
\begin{aligned}
\left|\frac{x}{\nu}-\lg \left(1+\frac{x}{\nu}\right)\right| & <\frac{1}{2} \frac{|x|^{2}}{\nu^{2}}\left\{1+\frac{2}{3} \cdot \frac{|x|}{\nu}+\frac{2}{4} \frac{|x|^{2}}{\nu^{2}}+\cdots\right\} \\
& <\frac{1}{2} \cdot \frac{|x|^{2}}{\nu^{2}}\left\{1+\frac{|x|}{\nu}+\frac{|x|^{2}}{\nu^{2}}+\cdots\right\} \\
& <\frac{1}{2} \frac{|x|^{2}}{\nu(\nu-|x|)}
\end{aligned}
$$

so hat. man für $m>|x|$

$$
\sum_{n}^{\infty}\left|\frac{x}{\nu}-\lg \left(1+\frac{x}{\nu}\right)\right|<\frac{|x|^{2}}{2} \sum_{m}^{\infty} \frac{1}{\nu \cdot(\nu-|x|)},
$$

sodass also die Reihe $\sum\left\{\frac{x}{\nu}-\lg \left(1+\frac{x}{\nu}\right)\right\}$ unbedingt convergirt für alle reellen oder complexen $x$ - mit Ausschluss der negativen ganzzahligen, weil für einen Werth $x$ von der Form $(-v)$ ein Glied der Reibe unendlich wird. Da hieraus insbesondere auch die Convergenz der Reihe für $x=1$ folgt, so wird man setzen können

$$
\sum_{1}^{\infty}\left\{\frac{1}{\nu}-\lg \left(1+\frac{1}{\nu}\right)\right\}=\lim _{n=\infty}\left\{\sum_{1}^{n} \frac{1}{\nu}-\lg (n+1)\right\}=C,
$$

wo $C$ einen bestimmten endlichen Werth hat und bekanntlich als die Euler'sche oder Mascheroni'sche Constante bezeichnet wird (man findet $C=0,577215 \ldots$...). Durch Einführung dieser Constanten $C$ nimmt die Gl. (10a) die Form an

$$
\lg \Gamma(x)=-\lg x+\sum_{1}^{\infty}\left\{\frac{x}{\nu}-\lg \left(1+\frac{x}{\nu}\right)\right\}-C x
$$

und aus ihrer unbedingten Convergenz (wobei zu den bereits ausgeschlossenen negativen ganzzahligen Werthen für $x$ jetzt auch noch der Werth $x=0$, wegen des Gliedes lg $x$, hinzukommt) folgt unmittelbar auch diejenige der ursprünglichen heihe (10), sowie des unendlichen Productes (9) bezw. (8). Zugleich ergiebt sich für letzteres aus $(10 \mathrm{~b})$ noch die weitere Form ${ }^{*}$ :

$$
\Gamma(x)=\frac{1}{e^{c x} \cdot x \prod_{1}^{\infty} \prod\left(1+\frac{x}{\nu}\right) e^{-\frac{x}{v}}}=\frac{1}{\mathcal{F}_{c}(x)},
$$

*) Die einzelnen Factoren sind hier Primfunctionen im Sinne des Herrn Weierstrass (Zur Theorie der eindeutigen analytischen Functionen - Art. 1). 
wenn man - nach Herrn Weierstrass' Vorgange für das im Nenner stehende, für $x=0,-1,-2, \cdots$ verschwindende, für alle übrigen endlichen $x$ gegen einen endlichen Werth unbedingt convergirende Product eine besondere Bezeichnung: Factorielle von $x$ einführt, so dass also

$$
F c(x)=e^{c x} x \prod_{1}^{\infty} \Gamma\left(1+\frac{x}{v}\right) e^{-\frac{x}{v}}=\frac{1}{\Gamma(x)}
$$

gesetzt wird.

Die Gültigkeit der in den Gleichungen (8) bis (11) enthaltenen Product- bezw. Reihenentwicklungen für $\Gamma(x)$ bezw. $\lg \Gamma(x)$ erstreckt sich ihrer Herleitung gemäss zunächst auf positive Werthe $x<1$. Die Gültigkeit für $x=1$ ergiebt sich ohne Weiteres aus Gl. (8) durch Vergleichung mit dem früher gefundenen Resultate $\Gamma(1)=1$. Sodann lässt sich aber zeigen, dass das unendliche Product in Gl. (8) - und folglich auch die damit identischen in Gl. (9) und (11) - bei Vermehrung des Argumentes um ganze Zahlen derselben Recursionsformel genügt, wie die $\Gamma$-Function. Setzt man etwa für den Augenblick

so wird

$$
\frac{1}{x} \lim \frac{1 \cdot 2 \cdot(n-1)}{(x+1)(x+2) \cdots(x+n-1)} n^{x}=P(x),
$$

$$
\begin{aligned}
P(x+1) & =\frac{1}{x+1} \lim \frac{1 \cdot 2 \cdots n-1}{(x+2)(x+3) \cdots(x+n)} n^{x+1} \\
& =\lim \frac{1 \cdot 2 \cdots n-1}{(x+1)(x+2) \cdots(x+n-1)} n^{x}\left(\frac{n}{x+n}\right)
\end{aligned}
$$

also wegen $\lim \left(\frac{n}{x+n}\right)=1$

und allgemein

$$
P(x+1)=x P(x)
$$

$$
P(x+n)=x(x+1) \cdots(x+n-1) \cdot P(x),
$$

woraus dann die Identität von $P(x)$ und $\Gamma(x)$, da sie für $0<x \leqq 1$ bereits feststeht, für alle positiven endlichen $x$ gefolgert werden kann. Das Product $P(x)$, dessen Convergenzgebiet, wie bemerkt, auch auf alle negativen und complexen endlichen Werthe von $x$ sich erstreckt, kann sodann dazu dienen, um die ursprünglich nur für positive $x$ definirte $\Gamma$-Function auf jenes erweiterte Gebiet fortzusetzen. (N.B. Die ursprüngliche Integraldefinition verliert ihren Sinn für alle negativen Werthe von $x$ und alle complexen mit negativem reellen Theil; für complexe $x$ mit positivem reellen Theil*) bleibt das Integral, wie sich leicht nachweisen lässt, bestimmt, endlich und gleich $P(x) * *$. -

*) Die Zulassung solcher Werthe findet sich zuerst in dem oben bereits eitirten Aufsatze von Poisson. (Journ. de l'Ecole polyt. Cah. 19, p. 477).

*) Vgl. Scheeffer, Ueber einige bestimmte Integrale, betrachtet als Functionen eines complexen Parameters. (Habilitationsschrift, 1883). 
Die durch Gl. (12) eingeführte Factorielle genügt - als reciproker Werth der $\Gamma$-Function - offenbar der Recursionsformel

sodass

$$
F_{c}(x+1)=\frac{1}{x} F_{c}(x),
$$

$$
F c(x+n)=\frac{F c(x)}{x \cdot(x+1) \cdots(x+n-1)} \quad F c(n+1)=\frac{1}{n !} .
$$

Man hat hiernach

und daher

$$
F c(1-x)=-\frac{1}{x} F c(-x)=e^{-c x} \prod_{1}^{\infty}\left(1-\frac{x}{v}\right)^{\frac{x}{v}}
$$

$$
F c(x) \cdot F c(1-x)=x \prod_{1}^{\infty} \Gamma\left(1-\frac{x^{2}}{v^{2}}\right)=\frac{\sin \pi x}{\pi},
$$

oder, wenn man statt der Factoriellen Г-Functionen einführt:

$$
\Gamma(x) \cdot \Gamma(1-x)=\frac{\pi}{\sin \pi x},
$$

eine weitere Hauptformel zur Berechnung der $\Gamma$-Functionen, mit deren Hülfe sich alle Argumente zwischen $\frac{1}{2}$ und 1 auf solche zwischen 0 und 1 reduciren lassen

Auch die sog. Legendre'sche Reihe d. h. die Potenzreihe für $\lg \Gamma(1+x)$ lässt sich aus den bisherigen Resultaten fast ohne Rechnung herleiten*). Es ist nämlich

$$
\lg \Gamma(1+x)=\lg \{x \cdot \Gamma(x)\}=\lg x+\lg \Gamma(x),
$$

also vach $\mathrm{Gl} .(10 \mathrm{~b})$

$$
\lg \Gamma(1+x)=-\lg x+\sum_{1}^{\infty}\left\{\frac{x}{\nu}-\lg \left(1+\frac{x}{\nu}\right) ! \cdot-C_{X}\right.
$$

Da $v \geqq 1$, so kann man unter der Voraussetzung, dass $|x|<1$ die Logarithmen nach Potenzen von $\frac{x}{v}$ entwickeln:

$$
\lg \Gamma(1+x)=-C x+\sum_{1}^{\infty}\left(\frac{1}{2} \frac{x^{2}}{\nu^{2}}-\frac{1}{3} \frac{x^{3}}{v^{3}}+\frac{1}{4} \frac{x^{4}}{v^{4}}-\cdots\right)
$$

und da die so entstehende Doppelreihe absolut convergirt - denn man hat ähnlich wie oben: Theorems :

**) Dagegen verzichte ich hier auf die Herleitung des sog. Gauss'schen

$$
\prod_{0}^{n-1} \Gamma\left(x+\frac{v}{n}\right)=(2 \pi)^{\frac{n-1}{2}} n^{-n x+\frac{1}{2}} \Gamma(n x)
$$

da dasselbe bereits von Ganss in der denkbar kürzesten Weise abgeleitet wurde (a. a. 0. Art. 26; vgl. auch: Serret, Diff.- und Int.-Rechn., Bd. II, Art. 528). 


$$
\begin{aligned}
& \sum_{1}^{\infty}\left(\frac{1}{2} \frac{|x|^{2}}{v^{2}}+\frac{1}{3} \frac{|x|^{3}}{v^{3}}+\frac{1}{4} \frac{|x|^{4}}{v^{4}}+\cdots\right) \\
< & \sum_{1}^{\infty} \frac{1}{2} \frac{|x|^{2}}{v^{2}}\left(1+\frac{|x|}{\nu}+\frac{|x|^{2}}{\nu^{2}}+\cdots\right) \\
< & \frac{|x|^{2}}{2} \sum_{1}^{\infty} \frac{1}{v(\nu-|x|)},
\end{aligned}
$$

so kann man sie nach Potenzen von $x$ ordnen, sodass sich ergiebt

(16) $\lg \Gamma(1+x)=-C x+\sum_{2}^{\infty}(-1)^{k} \frac{1}{k} \cdot S_{k} x^{k} \quad(|x|<1)$,

wenn

$$
\sum_{1}^{\infty} \frac{1}{v^{k}}=S_{k} \quad(k \geqq 2)
$$

gesetzt wird*). Da diese Reihe keine andere sein kann als die Maclaurin'sche, so folgt, dass:

$$
\begin{gathered}
\left(\frac{d \lg \Gamma(1+x)}{d x}\right)_{x=0}=\frac{\Gamma^{\prime}(1)}{\Gamma(1)}=\Gamma^{\prime}(1)=-C, \\
\left(\frac{d^{k} \lg \Gamma(1+x)}{d x^{k}}\right)_{x=0}=(-1)^{k}(k-1) ! S_{k} .
\end{gathered}
$$

Im übrigen hätte man diese letzten Gleichungen auch durch Differentiation der unbedingt und - wie leicht zu ersehen - auch gleichmässig convergenten Reihe (10) bezw. $(10 \mathrm{~b})$ herleiten können. Man erhält auf diese Weise die Beziehungen:

$$
\begin{aligned}
\frac{d \lg \Gamma(x)}{d x}=-\frac{1}{x} & +\sum_{1}^{\infty}\left\{\lg \left(1+\frac{1}{\nu}\right)-\frac{1}{x+\nu}\right\} \\
& =\sum_{1}^{\infty}\left\{\lg \left(1+\frac{1}{\nu}\right)-\frac{1}{x+\nu-1}\right\}
\end{aligned}
$$

(aus Gl. (10))

*) Die Reihe (16) ist für $x=+1-$ als alternirende Reihe mit beständig abnehmenden Gliedern - noch bedingt convergent und stellt dann - nach dem Abel-Dirichlet'schen Stetigkeitssatze - auch noch $\lg \Gamma(2)=0$ dar. In Folge dessen ergiebt sich die interessante Beziehung:

$$
C=\sum_{2}^{\infty} k(-1)^{k} \frac{1}{k} S_{k}=\frac{1}{2} S_{2}-\frac{1}{3} S_{3}+\frac{1}{4} S_{4}-\ldots
$$


$(20 \mathrm{~b})$

$$
\begin{aligned}
&=-\frac{1}{x}+ \sum_{1}^{\infty}\left\{\frac{1}{\nu}-\frac{1}{x+\nu}\right\}-C \\
&= \sum_{1}^{\infty}\left\{\frac{1}{\nu}-\frac{1}{x+\nu-1}\right\}-C \\
&(\text { aus Gl. }(10 \mathrm{~b}))
\end{aligned}
$$

und

$$
\frac{d^{k} \lg \Gamma(x)}{d x^{k}}=(-1)^{k}(k-1) ! \sum_{1}^{\infty} \frac{1}{(x+\nu-1)^{k}},
$$

welche für $x=1$ in die Gleichungen (18), (19) übergehen und in die Maclaurin'sche Formel für $\lg \Gamma(1+x)$ eingesetzt ebenfalls die Reihe (16) liefern*). Diese letztere kann man nun bekanntlich noch stärker convergent machen, indem man zunächst schreibt:

$\lg \Gamma(1+x)=-\lg (1+x)+(1-C) x+\sum_{2}^{\infty}(-1)^{k} \frac{1}{k}\left(S_{k}-1\right) x^{k}$ und hierauf die Reihe $\lg \Gamma(1-x)=-\lg (1-x)-(1-C) x+\sum_{2}^{\infty} \frac{1}{k}\left(S_{k}-1\right) x^{k}$ davon subtrahirt. Da nun:

$$
\Gamma(x) \Gamma(1-x)=\frac{1}{x} \Gamma(1+x) \cdot \Gamma(1-x)=\frac{\pi}{\sin \pi x}
$$

und daher :

$$
\lg \Gamma(1-x)=-\lg \Gamma(1+x)+\lg \frac{\pi x}{\sin \pi x},
$$

so erhält man schliesslich, nachdem man noch mit dem Factor 2 dividirt:

$$
\begin{aligned}
\lg \Gamma(1+x)=\frac{1}{2} \lg \frac{\pi x}{\sin \pi x} & -\frac{1}{2} \lg \frac{1+x}{1-x}+(1-C) x \\
& -\frac{1}{2} \sum_{1}^{\infty} \frac{1}{2 k+1}\left(S_{2 k+1}-1\right) x^{2 k+i}
\end{aligned}
$$

(für $|x|<1$ ) - als diejenige Reihe, welche man speciell als die Legendre'sche zu bezeichnen pflegt**).

*) Für ein ganzzahliges Argument $x=n$ ergiebt sich noch aus Gl. (20b):

$$
\frac{\Gamma^{\prime}(n)}{\Gamma(n)}=\frac{1}{1}+\frac{1}{2}+\cdots+\frac{1}{n-1}-C
$$

d. h. der logarithm. Differentialquotient von $\Gamma(x)$ ist für pos. ganzzablige $x$ durch eine endliche Reibe darstellbar.

**) Legendre, Traité des Fonctions Elliptiques et des Intégrales Eulériennes T. II, p. 433 , Art. 85. 


\section{$\S 2$.}

Das Gauss'sche und Dirichlet'sche Integral für $\frac{d \lg \Gamma(x)}{d x}$. Das Cauchy'sche Integral für $\lg \Gamma(x+1)$.

Die Gleichung (201) lässt sich auch folgendermassen schreiben:

$$
\begin{aligned}
\frac{d \lg \Gamma(x)}{d x} & =-\frac{1}{x}+\lim \sum_{1}^{n-1}\left\{\lg \left(1+\frac{1}{\nu}\right)-\frac{1}{x+\nu}\right\} \\
& =\lim \left\{\lg n-\sum_{0}^{x-1} \frac{1}{x+\nu}\right\} \quad(n=\infty) .
\end{aligned}
$$

Es möge jetzt wiederum $x$ reell und $>0$ angenommen werden.

Um nun zunächst $\lg n$ und $\sum_{0}^{n-1} \frac{1}{x+\nu}$ durch bestimmte Integrale auszudrücken, hat man aus der Identität:

$$
\frac{1}{z}=\int_{0}^{1} \alpha^{z-1} d \alpha
$$

wenn man dieselbe mit $d z$ multiplicirt und in den Grenzen 1 bis $n$ integrirt

$$
\left.\lg n=\int_{0}^{1} d \alpha \int_{1}^{n} \alpha^{z-1} d z=\int_{0}^{1} \frac{\alpha^{k-1}-1}{\lg \alpha} d \alpha^{*}\right)
$$

und andrerseits, wenn man statt $z \quad x+\nu$ schreibt und über den Index $\nu$ von 0 bis $n-1$ summirt

$$
\sum_{0}^{n-1} \frac{1}{\underset{x}{x+\nu}}=\int_{0}^{1} \sum_{0}^{n-1} v \alpha^{v+x-1} \cdot d \alpha=\int_{0}^{1} \frac{\alpha^{x-1}\left(1-\alpha^{2}\right)}{1-\alpha} d \alpha
$$

*) Dieses Integral nimmt, wenn man $e^{-\alpha}$ statt $\alpha$ und $x$ statt $n$ schreibt, die bek. Form an:

$$
\lg x=\int_{0}^{\infty} \frac{e^{-\alpha}-e^{-x \alpha}}{\alpha} d \alpha .
$$

Integrirt man diese fleichung, nachdem man mit $d x$ multiplicirt, nach $x$ in den Grenzen 1 und $x$, so wird:

$$
x(\lg x-1)+1=\int_{i}^{\infty}\left\{(x-1) e^{-\alpha}+\frac{e^{-x \alpha}-e^{-\alpha}}{\alpha}\right\} \frac{d \alpha}{\alpha},
$$

wie ich des Späteren wegen hier gleich bemerken will. 
Hieraus ergiebt sich aber:

$$
\begin{aligned}
\frac{d \lg \Gamma(x)}{d x} & =\lim _{n=\infty} \int_{0}^{\infty 1}\left\{\frac{\alpha^{n-1}-1}{\lg \alpha}-\frac{\alpha^{x-1}\left(1-\alpha^{n}\right)}{1-\alpha}\right\} d \alpha \\
& =\int_{0}^{1}\left\{\frac{1}{\left.\lg \frac{1}{\alpha}-\frac{\alpha^{x-1}}{1-\alpha}\right\} d \alpha-\lim _{n=\infty}} \int_{0}^{1} \alpha^{n-1}\left\{\frac{1}{\lg \frac{1}{\alpha}}-\frac{\alpha^{x}}{1-\alpha}\right\} d \alpha\right. \\
& =P+\lim _{n=\infty} R_{n} .
\end{aligned}
$$

Als möglicherweise kritische Stellen für die hierbei auftretenden Integralfunctionen sind lediglich die beiden Werthe $\alpha=0$ und $\alpha=1$ zu untersuchen. Von diesen scheidet indessen der Werth $\alpha=0$ sofort aus, da wegen $x>0 \quad \alpha^{x-1}$ (welches in $P$ vorkommt) von niedrigerer als der ersten Ordnung unendlich wird, also das betreffende Integral dadurch nicht alterirt wird, während $\alpha^{x}$ (in $R_{n}$ ) überhaupt nicht unendlich wird, sondern verschwindet. $U m$ ferner das Verhalten der zu integrirenden Functionen in der Nähe von $\alpha=1$ zu untersuchen, hat man, wenn $\varepsilon$ eine beliebig kleine positive Grösse bedeutet:

$$
\begin{aligned}
& \left(\frac{1}{\lg \frac{1}{\alpha}}\right)_{\alpha=1-\varepsilon}=(-\lg (1-\varepsilon))^{-1}=\varepsilon^{-1}\left(1+\frac{\varepsilon}{2}+\vartheta^{2}\right)^{-1} \\
& =\varepsilon^{-1}\left(1-\frac{\varepsilon}{2}+\vartheta^{\prime} \varepsilon^{2}\right), \\
& \left(\frac{\alpha^{x-1}}{1-\alpha}\right)_{\alpha=1-\varepsilon}=\varepsilon^{-1}(1-\varepsilon)^{x-1}=\varepsilon^{-1}\left(1+(x-1) \varepsilon+\frac{(x-1)(x-2)}{1 \cdot 2} \vartheta^{\prime \prime} \varepsilon^{2}\right) \\
& \left\{\frac{1}{\left.\lg \frac{1}{\alpha}-\frac{\alpha^{x-1}}{1-\alpha}\right\}_{\alpha=1-\varepsilon}=\left(\frac{1}{2}-x\right)+\left(\vartheta^{\prime}-\frac{(x-1)(x-2)}{2} \vartheta^{\prime \prime}\right) \varepsilon}\right.
\end{aligned}
$$

d. h. die betrachtete Function bleibt bei der Annäherung von $\alpha$ an den Werth 1 endlich, und zwar convergirt sie für $\alpha=1$ gegen den Werth $\frac{1}{2}-x$. Hieraus folgt aber zunächst, dass das Integral $P$ endlich und bestimmt ist.

Da ferner die soeben benützte Reihenentwicklung - wenn man dabei $x$ statt $x-1$ setzt - auch auf den in $R_{n}$ auftretenden Factor $\left\{\frac{1}{\lg \frac{1}{\alpha}}-\frac{\alpha^{x}}{1-\alpha}\right\}$ Bezug hat, so ergiebt sich, dass derselbe im ganzen Integrationsintervalle nirgends unendlich wird, sodass man - wenn $M$ einen endlichen Mittelwerth bezeichnet - setzen kann:

$$
R_{n}=M \int_{0}^{1} \alpha^{n-1} d \alpha=\frac{M}{n} \text {. }
$$

also

$$
\lim R_{n}=0 \quad(n=\infty) \text {. }
$$


Somit liefert die Gleichung (23) die folgende - soviel ich weiss, zuerst von Gauss abgeleitete*) Integral-Darstellung

$$
\frac{d \lg \Gamma(x)}{d x}=\int_{0}^{1}\left\{\frac{1}{\lg \frac{1}{\alpha}}-\frac{\alpha^{x-1}}{1-\alpha}\right\} d \alpha .
$$

Setzt man darin $\alpha$ statt $\lg \frac{1}{\alpha}$, also $e^{-\alpha}$ statt $\alpha$, so nimmt dieselbe die ebenfalls von Gauss**) gegebene Form an:

$$
\frac{d \lg \Gamma(x)}{d x}=\int_{0}^{\infty}\left\{\frac{e^{-\alpha}}{\alpha}-\frac{e^{-x \alpha}}{1-e^{-\alpha}}\right\} d \alpha
$$

Schliesslich ergiebt sich, wenn man den zweiten Bestandtheil dieses Integrals in der Weise transformirt, dass man $(1+\alpha)$ statt $e^{\alpha}$ setzt, die von Dirichlet herrührende Gleichung: ${ }^{* * *}$ )

$$
\begin{aligned}
\frac{d \lg \Gamma(x)}{d x} & =\int_{0}^{\infty}\left\{\frac{e^{-\alpha}}{\alpha}-\frac{1}{\alpha(1+\alpha)^{x}}\right\} d \alpha \\
& =\int_{0}^{\infty}\left\{e^{-\alpha}-(1+\alpha)^{-x}\right\} \frac{d \alpha}{\alpha} .
\end{aligned}
$$

Obschon die einzelnen Bestandtheile der Integrale (24b), (24c) wegen der unteren Grenze 0 divergent sind, so ist diese Transformation doch gestattet. Man kann nämlich zunächst, wenn $\delta$ beliebig klein gegeben wird, eine endliche Grösse $\varepsilon$ so bestimmen, dass

und

$$
\int_{0}^{e}\left\{\frac{e^{-\alpha}}{\alpha}-\frac{e^{-x \alpha}}{1-e^{-\alpha}}\right\} d \alpha
$$

$$
\int_{0}^{\varepsilon}\left\{\frac{e^{-\alpha}}{\alpha}-\frac{(1+\alpha)^{-x}}{\alpha}\right\} d \alpha
$$

absolut genommen kleiner als $\delta$ werden. Die Integrale

*) Ges. W. Bd. 3 S. 159, Gleichung [77]. Gauss setzt bekanntlich:

$$
\frac{d \lg \Pi(x)}{d x}=\frac{d \lg \Gamma(x+1)}{d x}=\Psi(x) \text {. }
$$

**) a. a. O. S. 160 , Gleichung [78].

***) Crelle's Journal, Bd. 15, S. 260, Gleichung 10. Dort ist auch die Znlässigkeit dieser Transformation (oder vielmehr der inversen, welche Gleichnng $(24 c)$ in (24b) überführt) besonders motivirt. 


$$
\int_{\varepsilon}^{\infty} \frac{e^{-\alpha}}{\alpha} d \alpha \int_{\varepsilon}^{\infty} \frac{e^{-x \alpha}}{1-e^{-\alpha}} d \alpha \int_{\varepsilon}^{\infty} \frac{(1+\alpha)^{-x}}{\alpha} d \alpha
$$

sind dann sämmtlich endlich und bestimmt. Man hat nun, wenn man in dem mittleren dieser Integrale $1+\alpha$ für $e^{\alpha}$ schreibt:

$$
\int_{e}^{\infty} \frac{e^{-x \alpha}}{1-e^{-\alpha}} d \alpha=\int_{e^{z}-1}^{\infty} \frac{(1+\alpha)^{-x}}{\alpha} d \alpha=\int_{\varepsilon+9 \varepsilon^{2}}^{\infty} \frac{(1+\alpha)^{-x}}{\alpha} d \alpha \quad(0<\vartheta<1)
$$

und daher

$$
\int_{\delta}^{\infty}\left\{\frac{e^{-\alpha}}{\alpha}-\frac{e^{-x \alpha}}{1-e^{-\alpha}}\right\} d \alpha=\int_{\varepsilon}^{\infty}\left\{\frac{e^{-\alpha}}{\alpha}-\frac{(1+\alpha)^{-x}}{\alpha}\right\} d \alpha+\int_{\varepsilon}^{\frac{e}{\left(1+\varepsilon^{2}\right.}} \frac{\int^{-x}}{\alpha} d \alpha
$$

oder da:

$$
\int_{\varepsilon}^{8+\vartheta \varepsilon^{2}} \frac{(1+\alpha)^{-x}}{\alpha} d \alpha<\vartheta \cdot \varepsilon^{2} \cdot \frac{(1+\varepsilon)^{-x}}{\varepsilon}<\vartheta \varepsilon
$$

mit $\varepsilon$ selbst beliebig klein wird, schliesslich:

$$
\int_{0}^{\infty}\left\{\frac{e^{-\alpha}}{\alpha}-\frac{e^{-x \alpha}}{1-e^{-\alpha}}\right\} d \alpha=\int_{0}^{\infty}\left\{\frac{e^{-\alpha}}{\alpha}-\frac{(1+\alpha)^{-x}}{\alpha}\right\} d \alpha,
$$

womit die Richtigkeit der Gleichung (24c) streng erwiesen ist.

Jedes der drei Integrale $(24 a, b, c)$ besteht aus zwei heterogenen und, einzeln genommen, divergenten Bestandtheilen: einer unendlich grossen Constanten und einer ebenfalls unendlich grossen Function von $x$. Die hier gegebene Ableitung dieser Integrale hat nun den Vorzug, dass sie die Provenienz dieser beiden Bestandtheile deutlich erkennen lässt: der constante Theil entspricht nämlich genau dem divergenten Bestandtheile $\sum_{1}^{\infty} \lg \left(1+\frac{1}{v}\right)$, der veränderliche dem ebenfalls divergenten Bestandtheile $\sum_{0}^{\infty} \frac{1}{x+v}$ in der Reihenentwicklung von $\frac{d \lg \Gamma(x)}{d x}$.

Setzt man in den Gleichungen $(24 \mathrm{a}, \mathrm{b}, \mathrm{c}) x=1$, so ergeben sich, da nach Gleichung (18) $\left(\frac{d \lg \Gamma(x)}{d x}\right)_{x=1}=\frac{\Gamma^{\prime}(1)}{\Gamma(1)}=\Gamma^{\prime}(1)=-C$, für die Euler'sche Constante "die Integralausdrücke 


$$
C=\left\{\begin{array}{l}
\int_{0}^{1}\left\{\frac{1}{1-\alpha}-\frac{1}{\lg \frac{1}{\alpha}}\right\} d \alpha, \\
\int_{0}^{\infty}\left\{\frac{1}{1-e^{-\alpha}}-\frac{1}{\alpha}\right\} e^{-\alpha} d \alpha, \\
\int_{0}^{\infty}\left\{(1+\alpha)^{-1}-e^{-\alpha}\right\} \frac{d \alpha}{\alpha} .
\end{array}\right.
$$

Durch Addition dieser Gleichungen zu den bezüglichen Gleichungen $(24 a, b, c)$ nehmen dieselben diejenige Form an, welche der Reihendarstellung

$$
\frac{d \lg \Gamma(x)}{d x}=\sum_{1}^{\infty}\left(\frac{1}{\nu}-\frac{1}{\nu+x-1}\right)-C(\mathrm{Gl} .(20 \mathrm{~b}))
$$

entspricht, und die man auch - analog wie oben - direct daraus herleiten könnte, nämlich:

$$
\frac{d \lg \Gamma(x)}{d x}=\left\{\begin{array}{l}
\int_{0}^{1} \frac{1-\alpha^{x-1}}{1-\alpha} d \alpha-C, \\
\int_{0}^{\infty} \frac{e^{-\alpha}-\alpha^{-x \alpha}}{1-e^{-\alpha}} d \alpha-C, \\
\int_{0}^{\infty}\left\{(1+\alpha)^{-1}-(1+\alpha)^{-x}\right\} \frac{d \alpha}{\alpha}-C .
\end{array}\right.
$$

Durch $(k-1)$-malige Differentiation der Gleichungen (26) ergiebt sich (für $k \geqq 2$ ):

$$
\begin{aligned}
\frac{d^{k} \lg \Gamma(x)}{d x^{k}}=-\int_{0}^{1} \frac{\lg ^{k-1} \alpha \cdot \alpha^{x-1}}{1-\alpha} d \alpha & =(-1)^{k} \int_{0}^{\infty} \frac{\alpha^{k-1} \cdot e^{-\alpha x}}{1-e^{-\alpha}} d \alpha \\
& =(-1)^{k} \int_{0}^{\infty} \frac{\lg k-1(1+\alpha)}{\alpha(1+\alpha)^{x}} d \alpha
\end{aligned}
$$

und hieraus für $x=1$, mit Berücksichtigung vou Gleichung (19): 


$$
\begin{aligned}
\sum_{1}^{\infty} \frac{1}{\nu^{k}} & =\frac{(-1)^{k-1}}{(k-1) !} \int_{0}^{1} \frac{\lg ^{k-1} \alpha}{1-\alpha} d \alpha \\
& =\frac{1}{(k-1) !} \int_{0}^{\infty} \frac{\alpha^{k-1}}{e^{\alpha}-1} d \alpha . \\
& =\frac{1}{(k-1) !} \int_{0}^{\infty} \frac{\lg ^{k-1}(1+\alpha)}{\alpha(1+\alpha)} d \alpha
\end{aligned}
$$

welche letzteren Gleichungen, sobald man darin $\Gamma(k)$ statt $(k-1)$ ! schreibt, übrigens nicht nur für ganzzahlige $k \geqq 2$, sondern für alle positiven $k>1$ gelten. Dies zeigt sich sofort, wenn man aus

bildet

$$
\Gamma(k)=\int_{0}^{\infty} e^{-\alpha} \cdot \alpha^{k-1} d \alpha=\nu^{k} \int_{0}^{\infty} e^{-\nu \alpha} \alpha^{k-1} d \alpha
$$

$$
\sum_{1}^{n} \frac{1}{v^{k}}=\frac{1}{\Gamma\left(\underset{c}{x)} \int_{0}\right.} \int_{0}^{\infty}\left(\alpha^{k-1} d \alpha \sum_{1}^{n} v e^{-v \alpha}\right)
$$

woraus sich unter der Voraussetzung, dass $k>1$, für $n=\infty$ in der That ergiebt

$$
\sum_{1}^{\infty} \frac{1}{\nu^{k}}=\frac{1}{\Gamma(k)} \int_{0}^{\infty} \frac{\alpha^{k-1} \cdot e^{-\alpha}}{1-e^{-\alpha}} d \alpha=\frac{1}{\Gamma(k)} \int_{0}^{\infty} \frac{\alpha^{k-1}}{e^{\alpha}-1} d \alpha .
$$

Ich übergehe die verschiedenen Integraldarstellungen für $\lg \Gamma(x)$, welche sich unmittelbar durch Integration der Gleichungen (24) und (26) nach $x$ ergeben.*) Hingegen will ich noch das Cauchy'sche Integral für $\lg \Gamma(x+1)$ hier ableiten, da sich die hierbei vorkommende Constantenbestimmung etwas kürzer und übersichtlicher gestalten lässt, wie dieselbe durch Cauchy und Stern gegeben worden ist.**) Schreibt man in Gleichung $(24 b) x+1$ statt $x$, so wird:

\section{oder:}

$$
\frac{d \lg \Gamma(k+1)}{d x}=\int_{0}^{\infty}\left\{\frac{e^{-\alpha}}{\alpha}-\frac{e^{-x \alpha-\alpha}}{1-e^{-\alpha}}\right\} d \alpha=\int_{0}^{\infty}\left\{\frac{e^{-\alpha}}{\alpha}-\frac{e^{-x \alpha}}{e^{\alpha}-1}\right\} d \alpha
$$

*) Cf. G. F. Meyer, Vorlesungen über best. Integrale, S. 131 .

**) Cauchy, Exerc. d'Analyse et de Physique Math., T. II, p. 386. Stern, in den Göttinger Studien, 1847, S. 306. - G. F. Meyer, a. a. O. S. 133 ff. - 


$$
\begin{aligned}
\frac{d \lg \Gamma(x+1)}{d x} & =\frac{1}{2} \int_{0}^{\infty} e^{-x \alpha} d \alpha+\int_{0}^{\infty} \frac{e^{-\alpha}-e^{-x \alpha}}{\alpha} d \alpha \\
& -\# \int_{0}^{\infty}\left\{\frac{1}{e^{\alpha}-1}-\frac{1}{\alpha}+\frac{1}{2}\right\} e^{-x \alpha} d \alpha \\
& =\frac{1}{2 x}+\lg x-\int_{0}^{\infty}\left\{\frac{1}{e^{\alpha}-1}-\frac{1}{\alpha}+\frac{1}{2}\right\} e^{-x \alpha} d \alpha
\end{aligned}
$$

und mithin, wenn man nach $x$ in den Grenzen 1 und $x$ integrirt:

$$
\begin{array}{r}
\lg \Gamma(x+1)=\frac{1}{2} \lg x+x(\lg x-1)+\int_{0}^{\infty}\left\{\frac{1}{e^{\alpha}-1}-\frac{1}{\alpha}+\frac{1}{2}\right\} \frac{e^{-x \alpha}}{\alpha} d \alpha \\
+1-\int_{0}^{\infty}\left\{\frac{1}{e^{\alpha}-1}-\frac{1}{\alpha}+\frac{1}{2}\right\} \frac{e^{-\alpha}}{\alpha} d \alpha .
\end{array}
$$

Um das constante Integral auszuwerthen setze man $x=\frac{1}{2}$, wobei zu beachten, dass:

$$
\Gamma\left(\frac{3}{2}\right)=\frac{1}{2} \Gamma\left(\frac{1}{z}\right)=\frac{1}{2} \sqrt{\pi}
$$

(da sich aus Gleichung (15) für $x=\frac{1}{2}$ ergiebt $\Gamma\left(\frac{1}{2}\right)^{2}=\pi$ ), und somit:

$$
\begin{array}{r}
\lg \frac{1}{2}+\frac{1}{2} \lg \pi=\frac{1}{2} \lg \frac{1}{2}+\frac{1}{2}\left(\lg \frac{1}{2}-1\right)+\int_{0}^{\infty}\left\{\frac{1}{e^{\alpha}-1}-\frac{1}{\alpha}+\frac{1}{2}\right\} \frac{e^{-\frac{\alpha}{2}}}{\alpha} d \alpha \\
+1-\int_{0}^{\infty}\left\{\frac{1}{e^{\alpha}-1}-\frac{1}{\alpha}+\frac{1}{2}\right\} \frac{e^{-\alpha}}{\alpha} d \alpha
\end{array}
$$

oder, wenn man in dem letzten Integrale $\frac{\alpha}{2}$ statt $\alpha$ einführt und $\frac{1}{e^{\frac{\alpha}{2}}-1}=\frac{e^{\frac{\alpha}{2}}+1}{e^{\alpha}-1}$ setzit:

$$
0=\frac{1}{2} \lg \pi-\frac{1}{2}+\int_{0}^{\infty}\left\{\frac{1}{e^{\alpha}-1}-\frac{e^{-\frac{\alpha}{2}}}{\alpha}\right\} \frac{d \alpha .}{\alpha}
$$

Addirt man diese Gleichung zu der oben gefandenen für $\lg \Gamma(x+1)$, so nimmt das constante Glied darin die Form an: 


$$
\begin{aligned}
& \frac{1}{2} \lg \pi+\frac{1}{2}+\int_{0}^{\infty}\left\{\frac{1-e^{-\alpha}}{e^{\alpha}-1}+\frac{e^{-\alpha}-e^{-\frac{\alpha}{2}}}{\alpha}-\frac{1}{2} e^{-\alpha}\right\} \frac{d \alpha}{\alpha} \\
= & \frac{1}{2} \lg \pi+\frac{1}{2}-\int_{0}^{\infty}\left\{-\frac{1}{2} e^{-\alpha}+\frac{e^{-\frac{\alpha}{2}}-e^{-\alpha}}{\alpha}\right\} \frac{d \alpha}{\alpha} .
\end{aligned}
$$

Das letzte Integral hat aber den Werth

$$
\frac{1}{2}\left(\lg \frac{1}{2}-1\right)+1=-\frac{1}{2} \lg 2+\frac{1}{2}
$$

(s. die Anmerkung zu Anfang dieses Paragraphen, wo in dem letzten Integral nur $x=\frac{1}{2}$ zu setzen ist), mithin ergiebt sich als Gesammtwerth dieser Constanten $\frac{1}{2} \lg \pi+\frac{1}{2} \lg 2=\frac{1}{2} \lg 2 \pi$, und man erhält schliesslich die Cauchy'sche Gleichung:

$$
\begin{aligned}
\lg \Gamma(x+1) & =\frac{1}{2} \lg 2 \pi x+x(\lg x-1) \\
& +\int_{0}^{\infty}\left\{\frac{1}{e^{\alpha}-1}-\frac{1}{\alpha}+\frac{1}{2}\right\} \frac{e^{-x \alpha}}{\alpha} d \alpha .
\end{aligned}
$$

Dieselbe liefert bekanntlich durch Einsetzen der Partialbruchentwicklung

$$
\frac{1}{e^{\alpha}-1}=\frac{1}{\alpha}-\frac{1}{2}+2 \sum_{1}^{\infty} \frac{\alpha}{\alpha^{2}+4 \nu^{2} \pi}
$$

die sogenannte Stirling'sche Reihe zur Berechnung von $\left.\lg \Gamma(x+1) .^{*}\right)$

\section{$\$ 3$.}

Andere Methode zur Herleitung der Productentwicklung für $\Gamma(x)$.

Den Ausgangspunkt dieser zweiten Methode bildet wiederum wie in $\$ 1$ - die Recursionsformel Gleichung (5):

$$
\Gamma(x)=\prod_{1}^{n} \frac{1}{x+v-1} \cdot \Gamma(x+n) .
$$

Während jedoch dort die weitere Entwicklung auf der Grenzbestimmung 'von $\Gamma(x+n)$ für $n=\infty$ beruhte, so soll hier die analoge Grenzbestimmung für das logarithmische Differential von $\Gamma(x+n)$ zu

*) Cf. G. F. Meyer, a. a. O. S. $141 \mathrm{ff}$. 
Grunde gelegt werden. Man hat zunächst, wenn man die obige Gleichung logarithmisch differenzirt:

$$
\frac{d \lg \Gamma(x)}{d x}=-\sum_{1}^{n} \frac{1}{x+\nu-1}+\frac{\Gamma^{\prime}(x+n)}{\Gamma(x+n)}
$$

Andererseits lässt sich das logarithmische Differential von $\Gamma(x)$ durch ein bestimmtes Integral darstellen: natürlich kann hierbei die im vorigen Paragraphen gegebene Ableitung dieser Integraldarstellung nicht benutzt werden, da dieselbe gerade auf der Productentwicklung für $\Gamma(x)$ beruhte. Man findet indessen nach Dirichlet*) aus:

$$
\Gamma^{\prime}(x)=\int_{0}^{\infty} e^{-\alpha} \cdot \alpha^{x-1} \lg \alpha \cdot d \alpha \quad(x>0)
$$

wenn man $\lg \alpha$ durch das gleichwerthige Integral $\int_{0}^{\infty} \frac{e^{-\beta}-e^{-\alpha \beta}}{\beta} d \beta$ ersetzt und die Integrationsfolge vertauscht**) - unmittelbar:

$$
\left.\Gamma^{\prime}(x)=\Gamma(x) \int_{0}^{\infty}\left\{e^{-\beta}-(1+\beta)^{-x}\right\} \frac{d \beta}{\beta} * * *\right)
$$

und wenn man im zweiten Theile dieses Integrales $1+\beta=e^{-\alpha}$, im ersten $\beta=\alpha$ setzt (was gestattet ist, wie im $\S 2$ gelegentlich der Gleichungen (24b), (24c) gezeigt wurde):

$\frac{\Gamma^{\prime}(x)}{\Gamma(x)}=\int_{0}^{\infty}\left\{\frac{e^{-\alpha}}{\alpha}-\frac{e^{-x \alpha}}{1-e^{-\alpha}}\right\} d \alpha=\lg x+\int_{0}^{\infty}\left\{\frac{1}{\alpha}-\frac{1}{1-e^{-\alpha}}\right\} e^{-x \alpha} d \alpha$.

Folglich hat man

$$
\frac{\Gamma^{\prime}(x+n)}{\Gamma(x+n)}=\lg (x+n)+\int_{0}^{\infty}\left\{\frac{1}{\alpha}-\frac{1}{1-e^{-\alpha}}\right\} e^{-(x+n) \alpha} d \alpha=\lg n+\varepsilon_{n}
$$

wo

$$
\varepsilon_{n}=\lg \left(1+\frac{x}{n}\right)+\int_{\alpha}^{\infty}\left\{\frac{1}{\alpha}-\frac{1}{1-e^{-\alpha}}\right\} e^{-(x+n) \alpha} d \alpha .
$$

*) Crelle's Journal, Bd. 15, S. 260.

**) Ueber die Zulässigkeit dieser Vertauschung s. Harnack, Elemente der Diff.- und Integr.-Rechnung, S. 300, \$162.

***) Bisher wurde diese Relation nur in der Weise für die Productentwicklung verwerthet, dass man daraus durch Differentiation einen Integralausdruck für $\frac{d^{2} \lg \Gamma(x)}{d x^{2}}$ herleitete, welcher nach Einführung einer neuen Integrationsvariablen eine Reihenentwicklung gestattet; aus dieser wird dann durch zweimalige Integration und geeignete Constantenbestimmung eine Reihe für $\lg \Gamma(x)$ gewonnen u. s. f. - vgl. Serret, Diff. und Integr.-Rechnung, Bd. II, Art. 521. - 
Nun ist leicht zu zeigen, dass $\varepsilon_{n}$ für hinlänglich grosse Werthe von $n$ beliebig klein gemacht werden kann. Zunächst hat man $($ da $x>0)$ :

$$
\lg \left(1+\frac{x}{n}\right)<\frac{x}{n}
$$

Da ferner $\frac{1}{\alpha}-\frac{1}{1-e^{-\alpha}}$ für keinen Werth $\alpha$ im ganzen Integrationsintervalle unendlich wird (auch nicht für $\alpha=0$ : denn

$$
\frac{1}{\alpha}-\frac{1}{1-e^{-\alpha}}=\frac{1}{\alpha}\left\{1-\frac{1}{1-\frac{\alpha}{2 !}+\frac{\alpha^{2}}{3 !}-\cdots}\right\}=\frac{1}{\alpha}\left\{\frac{\alpha}{2}+c \alpha^{2}+\cdots\right\}
$$

convergirt mit $\alpha$ stetig gegen $\frac{1}{2}$ ), so muss dieser Ausdruck innerhalb des Intervalles $(0 \ldots \infty)$ ein endliches, numerisches Maximum besitzen, dessen absoluter Werth mit $M$ bezeichnet werden möge. Alsdann wird aber:

$$
\text { abs. } \int_{0}^{\infty}\left\{\frac{1}{\alpha}-\frac{1}{1-e^{-\alpha}}\right\} e^{-(x+n) \alpha} d \alpha<M \int_{0}^{\infty} e^{-n \alpha} \cdot d \alpha<\frac{M}{n} .
$$

Daraus ergiebt sich, dass:

$$
\left|\varepsilon_{n}\right|<\frac{1}{n}(x+M)
$$

und, wie ich des folgenden wegen gleich bemerken will:

$$
\text { abs. } \int_{a}^{x} \varepsilon_{n} d x<\frac{1}{n}\left(\frac{x^{2}}{2}-\frac{a^{2}}{2}+M(x-a)\right)
$$

d. h. man hat für jedes endliche positive $x$

$$
\lim _{n=\infty} \varepsilon_{n}=0 \text { und } \lim _{n=\infty} \int_{a}^{x} \varepsilon_{n}^{x} d x=0 .
$$

Mit Hülfe der Beziehung (31) lässt sich jetzt Gleichung (30) folgendermassen schreiben:

$$
\frac{d \lg \Gamma(x)}{d x}=-\sum_{1}^{n} \frac{1}{x+\nu-1}+\lg n+\varepsilon_{n}
$$

oder, indem $\operatorname{man} n$ ins Unendliche wachsen lässt:

$$
\frac{d \lg \Gamma(x)}{d x}=\lim _{n=\infty}\left\{-\sum_{1}^{n} \frac{1}{x+v-1}+\lg n\right\} \text {. }
$$

Integrirt man ferner Gleichung (32) nach $x$ in den Grenzen 1 und $x$, so wird

$$
\lg \Gamma(x)=\sum_{1}^{n} \cdot\left\{\lg v-\lg (x+v-1\}+(x-1) \lg n+\int_{1}^{x} \varepsilon_{n} d x\right.
$$


oder auch, für $n=\infty$ :

d. h.

$$
\lg \Gamma(x)=\lim _{n=\infty}\left[\sum_{1}^{n}\{\lg \nu-\lg (x+\nu-1)\}+(x-1) \lg n\right]
$$

$$
\begin{aligned}
\Gamma(x)=\lim _{n=\infty}\left\{\prod_{1}^{n} \frac{\nu}{x+\nu-1} n^{x-1}\right\} & =\frac{1}{x} \lim _{n=\infty}\left\{\prod_{1}^{n-1} \frac{\nu}{\nu+x} n^{x}\right\} \\
& =\frac{1}{x} \prod_{1}^{\infty} \frac{\left(1+\frac{1}{v}\right)^{x}}{1+\frac{x}{v}} .
\end{aligned}
$$

Man kann auch die rechte Seite der Gleichung (32) von vornherein so umformen, dass sie für $n=\infty$ die Form einer unbedingt und gleichmässig convergirenden, also gliedweise integrirbaren Reihe annimmt. Man hat zunächst

$$
\frac{d \lg \Gamma(x)}{d x}=-\frac{1}{x}-\sum_{1}^{n-1}\left(\frac{1}{x+\nu}-\frac{1}{\nu}\right)+\left(\lg n-\sum_{1}^{n-1} \frac{1}{\nu}\right)+\varepsilon_{n}
$$

und wenn man wiederum (wie in Gleichung (11))

setzt:

$$
\begin{aligned}
\lim _{n=\infty}\left\{\sum_{1}^{n-1} \frac{1}{\nu}-\lg n\right\} & =\lim _{n=\infty} \sum_{1}^{n-1}\left\{\frac{1}{\nu}-\lg \left(1+\frac{1}{\nu}\right)\right\} \\
& =\sum_{1}^{\infty}\left\{\frac{1}{\nu}-\lg \left(1+\frac{1}{\nu}\right)\right\}=C
\end{aligned}
$$

$$
\frac{d \lg \Gamma(x)}{d x}=-C-\frac{1}{x}-\sum_{1}^{\infty}\left(\frac{1}{x+\nu}-\frac{1}{\nu}\right),
$$

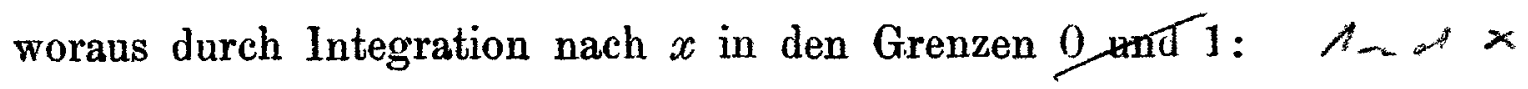

$$
\begin{aligned}
\lg \Gamma(x)=-(x-1) C-\lg x-\sum_{1}^{\infty}\left\{\lg (\nu+x)-\lg (\nu+1)-(x-1) \frac{1}{\nu}\right\} \\
=-(x-1) C-\lg x-\sum_{1}^{\infty}\left\{\lg (\nu+x)-\lg \nu-\frac{x}{\nu}\right\} \\
-\sum_{1}^{\infty}\left\{\frac{1}{\nu}-\lg \left(1+\frac{1}{\nu}\right)\right\} \\
=-C x-\lg x-\sum_{1}^{\infty}\left\{\lg \left(1+\frac{x}{\nu}\right)-\frac{x}{\nu}\right\}
\end{aligned}
$$

und daher 


$$
\Gamma(x)=\frac{1}{e^{c x} x \prod_{1}^{\infty} \prod\left(1+\frac{x}{v}\right) e^{-\frac{x}{v}}}
$$

übereinstimmend mit Gleichung (12). -

\section{$\S 4$.}

Das Euler'sche Integral erster Gattnng.

Das Euler'sche Integral erster Gattung

$$
\left.B(x, y)=\int_{0}^{\infty} \frac{\alpha^{x-1}}{(1+\alpha)^{x+y}} d \alpha^{*}\right)
$$

ist endlich und bestimmt, sobald $x>0, y>0$, und nimmt durch die Substitutionen $x=\frac{\beta}{1-\beta}, \beta=1-\gamma-$ wenn statt $\beta$ und $\gamma$ schliesslich wieder $\alpha$ geschrieben wird, die Formen an:

$$
\left\{\begin{aligned}
\text { (a) } B(x, y) & =\int_{0}^{1} \alpha^{x-1}(1-\alpha)^{y-1} d \alpha \\
\text { (b) } & =\int_{0}^{1} \alpha^{y-1}(1-\alpha)^{x-1} d \alpha=B(y, x)=\int_{0}^{\infty} \frac{\alpha^{y-1}}{(1+\alpha)^{x+y}} d \alpha .
\end{aligned}\right.
$$

Schreibt man:

$$
\begin{aligned}
B(x, y) & =\int_{0}^{1} \alpha^{x-1}(1-\alpha)^{y-1}(1-\alpha+\alpha) d \alpha- \\
& =B(x, y+1)+B(x+1, y)
\end{aligned}
$$

und bemerkt, dass:

$$
\begin{aligned}
B(x+1, y) & =\int_{U}^{!} \alpha^{x}(1-\alpha)^{y-1} d \alpha \\
& =\left[\frac{-\alpha^{x}(1-\alpha)^{y}}{y}\right]_{0}^{1}+\frac{x}{y} \int_{0}^{\frac{1}{3}} \alpha^{x-1}(1-\alpha)^{y} d \alpha \\
& =\frac{x}{y} B(x, y+1)
\end{aligned}
$$

so ergiebt sich die fundamentale Recursionsformel:

$$
B(x, y)=\frac{x+y}{y} B(x, y+1)=\frac{x+y}{x} B(x+1, y) .
$$

*) Die Bezeichnnng $\boldsymbol{B}(x, y)$ stammt von Binet: Mémoire sur les Intégrales Eulériennes. (Journal de l'École polyt. Cah. 27, p. 131). Legendre bezeichnet das betreffende Integral mit $(x, y)$. 
Durch n-malige Anwendung dieser Gleichung erhält man:

$$
\begin{aligned}
B(x, y) & =\frac{(x+y)(x+y+1) \cdots(x+y+n-1)}{y} \cdot B(x, y+n) \\
& =\frac{x+y}{y} \prod_{1}^{n-1} \frac{x+y+v}{y+v} \cdot B(x, y+n) .
\end{aligned}
$$

Um aus dieser Gleichung eine ünendliche Productentwicklung für $B(x, y)$ zu gewinnen, hat man nur das Verhalten von $B(x, y+n)$ für unbegrenzt wachsende $n$ zu untersüchen.

Da nun aus der definirenden Gleichung (34a) ohne weiteres folgt, dass für jedes positive $h$

$$
B(x, y)>B(x, y+h),
$$

so hat man, wenn $p$ die kleinste ganze Zahl bedeutet, die $\geqq y$ ist:

oder:

$$
B(x, n)>B(x, y+n)>B(x, p+n)
$$

$$
1>\frac{B(x, y+n)}{B(x, n)}>\frac{B(x, p+n)}{B(x, n)} \text {. }
$$

Nun folgt aus Gleichung (36), wenn man dieselbe umkehrt und $y=1$, $n=m-1$ setzt:

$$
B(x, m)=\frac{1.2 \ldots(m-1)}{(x+1)(x+2) \ldots(x+m-1)} B(x, 1)
$$

oder, da sich unmittelbar

ergiebt:

$$
B(x, 1)=\int_{0}^{1} \alpha^{x-1} d \alpha=\frac{1}{x}
$$

$$
B(x, m)=\frac{1}{x} \prod_{1}^{m-1} \frac{v}{x+v} .
$$

Mit Benutzung dieses Resultates geht die oben gefundene Ungleichung über in:

$$
\begin{aligned}
1>B(x, y+n) \cdot x \prod_{1}^{n-1} \frac{x+\nu}{\nu}> & \frac{n \cdot(n+1) \ldots(n+p-1)}{(x+n)(x+n+1) \ldots(x+n+p-1)} \\
> & \left(\frac{n}{x+n}\right)^{p}=\left(1+\frac{x}{n}\right)^{-p}=1-\vartheta \frac{p x}{n} \\
& (0<\vartheta<1),
\end{aligned}
$$

sodass man setzen kann

$$
B(x, y+n) \cdot x \prod_{1}^{n-1} \frac{x+\nu}{\nu}=1-\varepsilon_{n},
$$


wo

also

$$
\varepsilon_{n}=\vartheta^{\prime} \frac{p x}{n} \quad\left(0<\vartheta^{\prime}<\vartheta<1\right)
$$

$$
\lim \varepsilon_{n}=0(n=\infty) \text {. }
$$

Mithin lässt sich jetzt Gleichung (36) folgendermassen schreiben:

$$
B(x, y)=\frac{x+y}{x y} \prod_{1}^{n-1} \frac{\nu \cdot(x+y+\nu)}{(x+\nu)(y+\nu)}\left(1-\varepsilon_{n}\right)
$$

oder auch, indem man $n$ ins Unendliche wachsen lässt:

(39a) $B(x, y)=\frac{x+y}{x y} \prod_{1}^{\infty} \frac{v(x+y+v)}{(x+\nu)(y+\nu)}=\frac{x+y}{x y} \prod_{!}^{\infty} \frac{\left(1+\frac{x+y}{v}\right)}{\left(1+\frac{x}{v}\right)\left(1+\frac{y}{v}\right)}$.

Die Convergenz dieses Productes erkennt man auf Grund des in $\S 1$ gesagten unmittelbar, wenn man dasselbe in die Form setzt:

(39b) $B(x, y)=\frac{x+y}{x y} \frac{\prod\left(1+\frac{x+y}{v}\right) e^{-\frac{x+y}{v}}}{\prod\left(1+\frac{x}{\nu}\right) e^{-\frac{x}{v}} \prod\left(1+\frac{y}{\nu}\right) e^{-\frac{y}{\nu}}}(\nu=1 \cdots \infty)$ wo dann jedes dieser Theilproducte einzeln unbedingt convergirt und zwar nicht nur für positive Werthe von $x$ und $y$, sondern auch für alle negativen und complexen, sofern nicht gerade $x$ oder $y$ oder $(x+y)$ der Null oder einer ganzen negativen Zahl gleich wird. (NB. Das Product hat den Werth $\infty$, wenn mindestens eine der Variablen $x, y$ verschwindet oder negativ ganzzahlig wird; es hat den Werth Null, wenn das analoge lediglich von der Summe $(x+y)$ gilt). Die Gleichung (39b) bez. (39a) kann hiernach wiederum - analog wie die Productentwicklung für $\Gamma(x)$ - als erweiterte Definition der Function $B(x, y)$ angesehen werden. Zugleich ergiebt sich aus Gleichung $(39 \mathrm{~b})$ durch Vergleichung mit (12) (13) die wichtige Beziehung

$$
B(x, y)=\frac{\Gamma(x) \Gamma(y)}{\Gamma(x+y)}=\frac{F c(x+y)}{F c(x) F(y)} .
$$

Setzt man speciell $y=1-x$, so wird mit Berücksichtigung von Gleichung (15):

$$
B(x, 1-x)=\frac{\pi}{\sin \pi x}
$$

(für beliebige complexe $x$ )

und falls $x$ und $(1-x)$ beide positiv, d. h. $x$ ein positiver echter Bruch:

$$
\int_{0}^{1} \frac{\alpha^{x-1}}{1+\alpha} d \alpha=\int_{0}^{1} \frac{\alpha^{-x}}{1+\alpha} d \alpha=\frac{\pi}{\sin \pi x} \quad(0<x<1) .
$$


Bezeichnet ferner $n$ eine positive garize Zahl, so folgt aus Gleichung (40) mit Berücksichtigung von Gleichung (6)

$$
\Gamma(x)=\frac{\Gamma(x+n)}{(n-1) !} B(x, n)=\left(1+\vartheta \varepsilon_{n}\right) \cdot n^{x} B(x, n)
$$

oder auch:

$$
\Gamma(x)=\lim n^{x} B(x, n)
$$

eine Relation, die sich für $x>0$ auch an den betreffenden Integraldefinitionen verificiren lässt und alsdann zur Ableitung der Productentwicklung von $\Gamma(x)$ aus derjenigen von $B(x, n)$ dienen kann.*) Berlin, October 1887.

*) Cf. G. F. Meyer, a. a. 0. § 35-37, S. $97 \mathrm{ff}$. 